INPLASY

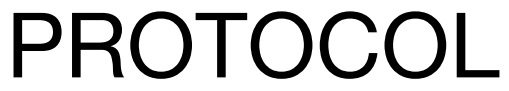

To cite: Rao et al. The comparison of different acupuncture therapies for post stroke depression: a Bayesian network meta-analysis protocol. Inplasy protocol 2020100104. doi:

10.37766/inplasy2020.10.0104

Received: 27 October 2020

Published: 27 October 2020

Corresponding author: Yuzheng Du

drduyuzheng@163.com

Author Affiliation:

First Teaching Hospital of Tianjin University of Traditional Chinese Medicine; National Clinical Research Center for Chinese Medic

Support: NSFC(NO: 81904093).

Review Stage at time of this submission: The review has not yet started.

Conflicts of interest: The authors have no conflicts of interest to disclose.

\section{The comparison of different acupuncture therapies for post stroke depression: a Bayesian network meta-analysis protocol}

Rao, C1; Liu, W2; Li, ZF3; Nan, X4; Yin, CS5; Yang, JP6; Du, YZ7.

Review question / Objective: Patients who suffered post stroke depression The RCTs with experiment group(EG) and control group(CG) meeting one of the following conditions will be included: (1) the intervention of EG is the therapy based on acupuncture(including acupuncture alone, or the acupuncture plus fluoxetine), the intervention of CG is fluoxetine; (2) using acupuncture plus placebo as the intervention of EG, and fluoxetine plus sham acupuncture as the or the intervention of CG (3) using different kinds of acupuncture therapies as the intervention of CG and EG respectively. Primary outcomes Degree of reduction in depression, measured by self-rating scales such as Beck Depression Inventory (BDI) or nurseadministered rating scale such as Hamilton Depression Rating Scale (HAMD). Total numbers of adverse events Secondary outcomes Quality of life indices such as the World Health Organization Quality of Life (WHOQOL). Improvement of social and life functions such as Activity of Daily Living Scale (ADL) The expression of nerve cell factors, such as serotonin (5-HT), IL-23. There are several scales used for assessment of depression, for example HAMA, MADRS, SDS, $\mathrm{BDI}$, we treat all scales equally.

INPLASY registration number: This protocol was registered with the International Platform of Registered Systematic Review and Meta-Analysis Protocols (INPLASY) on 27 October 2020 and was last updated on 27 October 2020 (registration number INPLASY2020100104).

\section{INTRODUCTION}

Review question / Objective: Patients who suffered post stroke depression The RCTs with experiment group(EG) and control group(CG) meeting one of the following conditions will be included: (1) the intervention of EG is the therapy based on acupuncture(including acupuncture alone, or the acupuncture plus fluoxetine), the intervention of CG is fluoxetine; (2) using acupuncture plus placebo as the 
intervention of EG, and fluoxetine plus sham acupuncture as the or the intervention of CG (3) using different kinds of acupuncture therapies as the intervention of $C G$ and $E G$ respectively. Primary outcomes Degree of reduction in depression, measured by self-rating scales such as Beck Depression Inventory (BDI) or nurse-administered rating scale such as Hamilton Depression Rating Scale (HAMD). Total numbers of adverse events Secondary outcomes Quality of life indices such as the World Health Organization Quality of Life (WHOQOL). Improvement of social and life functions such as Activity of Daily Living Scale (ADL) The expression of nerve cell factors, such as serotonin (5-HT), IL-23. There are several scales used for assessment of depression, for example HAMA, MADRS, SDS, BDI, we treat all scales equally.

Condition being studied: Depression is a common disease which occurs after stroke, affecting approximately one third of stroke survivors at any one time after stroke (compared with $5 \%-13 \%$ of adults without stroke), with a cumulative incidence of $55 \%$. Acupuncture, which has a long history in China, is the generic name of different kinds of acupuncture therapies, including manual acupuncture (MA), electroacupuncture(EA), fire needle(FN), dry needling(DN) and so on. Clinical studies have shown that acupuncture has a good therapeutic effect on post stroke depression (PSD), but the evidence-based medicine of it is insufficient The purpose of this study is to systematically evaluate the efficacy of different kinds of acupuncture therapies in the treatment of PSD, and to provide evidence-based basis for the clinical application of acupuncture in the treatment of PSD.

\section{METHODS}

Search strategy: PubMed, The Cochrane Library, Medline, Embase, China National Knowledge Infrastructure (CNKI), WanFang Data, VIP and Chinese Biomedical Databases. \#1 Stroke [Title/Abstract] \#2 Apoplexy [Title/Abstract] \#3 Cerebral Hemorrhage [Title/Abstract] \#4 Cerebrum
Hemorrhage [Title/Abstract] \#5 Cerebral Infarction [Title/Abstract] \#6 Cerebral Infarct [Title/Abstract] \#7 \#1 OR \#2 OR \#3 OR \#4 OR \#5 OR \#6 \#8 Acupuncture [Title/ Abstract] \#9 Electroacupuncture [Title/ Abstract] \#10 Laser Needle [Title/Abstract] \#11 Fire Needle [Title/Abstract] \#12 Dry Needling [Title/Abstract] \#13 \#8 OR \#9 OR \#10 OR \#11 OR \#12 \#14 Depression [Title/ Abstract] \#15 Depressive [Title/Abstract] \#16 \#14 OR \#15 \#17 \#7 AND \#13 AND \#16.

Participant or population: We will include RCTs that involved patients who suffered post stroke depression. The diagnosis of stroke should base on computer tomography (CT), magnetic resonance imaging (MRI), or clinical criteria. Meanwhile, depression were diagnosed according to the International Classification of Diseases, Tenth Edition (ICD-10), the Diagnosis and Statistical Manual of Mental Disorders (DSM)19, Chinese Classification of Mental Disorders (CCMD) or the 17-item Hamilton Rating Scale for Depression (HAMD-17).

Intervention: (1) the intervention of experiment group is the therapy based on acupuncture(including acupuncture alone, or the acupuncture plus SSRIs); (2) Using acupuncture plus placebo as the intervention of experiment group; (3) Using different kinds of acupuncture therapies as the intervention of experiment group.

Comparator: (1)The intervention of control group is SSRIs; (2)SSRIs plus sham acupuncture as the or the intervention of control group (3)Using different kinds of acupuncture therapies as the intervention of control group.

Study designs to be included: We will include RCTs that involved patients who suffered post stroke depression. The diagnosis of stroke should base on computer tomography (CT), magnetic resonance imaging (MRI), or clinical criteria. Meanwhile, depression were diagnosed according to the International Classification of Diseases, Tenth Edition (ICD-10), the Diagnosis and Statistical Manual of Mental Disorders (DSM)19, 
Chinese Classification of Mental Disorders (CCMD) or the 17-it.

Eligibility criteria: The RCTs with experiment group(EG) and control group(CG) meeting one of the following conditions will be included: (1) the intervention of EG is the therapy based on acupuncture(including acupuncture alone, or the acupuncture plus SSRIs), the intervention of CG is SSRIs; (2) using acupuncture plus placebo as the intervention of EG, and SSRIs plus sham acupuncture as the or the intervention of CG (3) using different kinds of acupuncture therapies as the intervention of CG and EG respectively. Our selection of acupuncture therapy for analysis will include MA, EA, $F N$, DN, laser needle(LN), or the combination of different kinds of acupuncture therapies, regardless of acupoint selection or acupuncture manipulation. According to our preliminary search result in the database, we found that there were few trials focused on the comparison between different kinds of acupuncture therapies, whereas more trials were focused on the comparison between acupuncture and western medicine.

Information sources: The following databases will be searched from inception to July 2020: PubMed, The Cochrane Library, Medline, Embase, China National Knowledge Infrastructure (CNKI), WanFang Data, VIP and Chinese Biomedical Databases.

Main outcome(s): (1) Degree of reduction in depression, measured by self-rating scales such as Beck Depression Inventory (BDI) or clinician-rated rating scale such as Hamilton Depression Rating Scale (HAMD); (2) Total numbers of adverse events.

Additional outcome(s): (1) Quality of life indices such as the World Health Organization Quality of Life (WHOQOL); (2) Improvement of social and life functions such as Activity of Daily Living Scale (ADL); (3) The expression of nerve cell factors, such as serotonin (5-HT), IL-23.
Data management: NoteExpress V.3.2 will be used to manage studies. First, duplicate literature will be removed through electronic/manual-based steps in NE. Second, two reviewers will independently screen the study article titles and abstracts and select the studies which meet the eligibility criteria. If there are disagreements, the third reviewer should be consulted. Two reviewers independently extracted the general information of the included studies. Extracted data include authors, year of publication, sample size, source of diagnosis, the observation period, interventions and test results of all groups. If there is any necessary data missing, corresponding authors will be contacted and asked to provide relevant details. Some studies will be excluded if the full text or data are unable to get access and the reasons for exclusion will be reported in detail in these cases.

Quality assessment / Risk of bias analysis: According to Cochrane Handbook for Systematic Reviews of Interventions version 6(https://training.cochrane.org/ handbook/current/chapter-08), the methodological quality and the risk of bias of the included studies by means of the risk of bias 2.0(ROB 2.0) tool. One researcher assessed the risk of bias of included studies by using ROB 2.0 and the other researcher confirmed the judgment. Any differences will be resolved through discussions with the third researcher. The following items were categorized as having high, low or unclear risk of bias: random sequence generation, allocation concealment, blinding of participants and personnel, blinding of outcome assessment, incomplete outcome data, selective reporting, and other biases which focusing on baseline imbalance.

Strategy of data synthesis: Bayesian network analysis will be conducted to compare the effects of different acupuncture therapies. STATA V.14.0 and ADDIS V.1.16.7 will be used to perform the network meta-analysis. We'll use the 12 statistic will to assess the heterogeneity. If the 12 value is below $50 \%$, the fixed effect model will be used. Otherwise, sensitivity 
analysis and subgroup analysis will be conducted to explore the main sources of heterogeneity, after which, the random effect model will be used if the 12 is still equal or greater than $50 \%$. Both types of effect sizes will be presented with $95 \% \mathrm{Cls}$, and values of $p<0.05$ will be regarded as statistically significant. Continuous outcomes will be calculated as mean differences (MDs) or standardized mean differences (SMDs), meanwhile, binary outcomes will be calculated as odds ratios (ORs).

Subgroup analysis: If the 12 value is equal

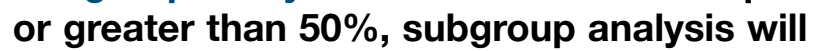
be conducted to explore the main sources of heterogeneity.The grouping will base on the results of literature search.

Sensibility analysis: If the 12 value is equal or greater than $\mathbf{5 0 \%}$, sensitivity analysis will be conducted to explore the main sources of heterogeneity, after which, the random effect model will be used if the $I 2$ is still equal or greater than $50 \%$. Both types of effect sizes will be presented with $95 \%$ Cls, and values of $p<0.05$ will be regarded as statistically significant.

Country(ies) involved: China.

Keywords: network meta-analysis; acupuncture; protocol; post stroke depression.

Contributions of each author:

Author 1 - Chang Rao - The author did the data curation, drafted and review the manuscript.

Author 2 - Wei Liu - The author did the data curation, drafted and review the manuscript.

Author 3 - Zefang Li - The author contributed to the resources and software.

Author 4 - Xi Nan - The author contributed to the resources and software.

Author 5 - Chunsheng Yin - The author contributed to the software.

Author 6 - Jipeng Yang - The author contributed to the investigation.

Author 7 - Yuzheng Du - The author read, provided feedback. 